\title{
Use of cardiovascular computed tomography in the diagnosis and management of coarctation of the aorta
}

\author{
Matthew J. Budoff, MD, Adekunle Shittu, MD, and Sion Roy, MD
}

\begin{abstract}
Coarctation of the aorta is a common malformation accounting for $5 \%$ to $8 \%$ of all congenital heart defects. Although echocardiography and magnetic resonance imaging are most frequently used in the diagnosis and management of coarctation of the aorta, the role of multidetector computed tomography in congenital heart defects has been expanding and its use has been increasing over the last few years. Multidetector computed tomography with its high spatial and temporal resolution, multiplanar reconstruction capabilities, and wide field of view is an excellent tool for the detection and characterization of coarctation of the aorta, and the identification of associated anomalies of the heart and pulmonary vasculature. It is particularly useful and indicated in patients with contraindications for cardiac magnetic resonance (eg, patients with aortic or coronary artery stents, pacemakers or implantable cardioverter defibrillators, and mechanical heart valves). Knowledge of the cardiac computed tomography appearance of coarctation of the aorta and associated abnormalities is critical for accurate diagnosis and management, which includes providing information to plan surgical or percutaneous therapy. We present various forms of coarctation of the aorta on cardiac computed tomography. (J Thorac Cardiovasc Surg 2013;146:229-32)
\end{abstract}

With advances in the use of cardiovascular imaging in the management of coarctation of the aorta, cardiac computed tomography (CCT) has emerged as an excellent imaging modality. CCT has the highest resolution (spatial and temporal) among the noninvasive imaging modalities available for analyzing the cardiovascular system in the diagnosis and management of diseases of the aorta. ${ }^{1}$

Advantages of CCT include providing a simultaneous visualization of the ascending aorta, aortic arch, descending aorta, and aortic valve; preoperative detailed structural information as guidance for corrective procedures/surgeries; simultaneous information about coronary artery course (anomalies) and disease; and a short acquisition time for images. Although radiation dose is of continued concern, doses have decreased by $80 \%$ with the advent and use of prospective triggering. ${ }^{2}$

Although cardiac magnetic resonance holds significant advantages in regard to radiation and measurements of pressures/gradients, CCT has several imaging advantages in this congenital heart disease population. With CCT, there is no claustrophobia, no difficulty for patients with congestive heart failure/orthopnea because imaging times are short,

\footnotetext{
From the Division of Cardiology, Los Angeles Biomedical Research Institute at Harbor-UCLA, Torrance, Calif.

Disclosures: Dr Budoff is on the speakers' bureau for GE. The other authors have nothing to disclose with regard to commercial support.

Received for publication Oct 2, 2012; revisions received Dec 17, 2012; accepted for publication Jan 14, 2013; available ahead of print Feb 11, 2013.

Address for reprints: Matthew J. Budoff, MD, Los Angeles Biomedical Research Institute, 1124 West Carson St, Torrance, CA 90502 (E-mail: mbudoff@labiomed. org).

$0022-5223 / \$ 36.00$

Copyright (C) 2013 by The American Association for Thoracic Surgery

http://dx.doi.org/10.1016/j.jtcvs.2013.01.024
}

no signal void artifact associated with prior transcatheter interventions, and no contraindication in patients with implantable cardioverter defibrillators or pacemakers or in patients with aortic or coronary artery stents.

When compared with transthoracic echocardiography, CCT holds no difficulty in patients with chest wall deformities (eg, pectus deformities); has good acoustic windows; provides excellent evaluation of the transverse aorta, great vessels, and arch; and has no sedation risks.

Because coarctation of the aorta is diagnosed in many patients in childhood, CCT angiography can still be a relatively safe and useful modality, because the newer methods of radiation dose reduction can cumulatively reduce the estimated radiation dose by $90 \%$ without compromising image quality or diagnostic accuracy. ${ }^{2}$ Multiple studies have demonstrated similar or superior radiation reduction.

\section{COARCTATION OF THE AORTA}

Coarctation of the aorta is typically a discrete narrowing of the thoracic aorta just distal to the origin of the left subclavian artery. In some cases constriction may be proximal to the left subclavian artery, rarely in the abdominal aorta, and sometimes presents as a long segment or a tubular hypoplasia.

Coarctation of the aorta accounts for $5 \%$ to $8 \%$ of all congenital heart defects, occurring 4 times more frequently in male patients than in female patients. ${ }^{3}$ There is a frequent association with a bicuspid aortic valve, with a reported incidence of $30 \%$ to $40 \%{ }^{4}$ Other associated abnormalities include ventricular septal defect, patent ductus arteriosus, aortic stenosis, and parachute mitral stenosis. ${ }^{5}$ The most important noncardiac abnormality is 

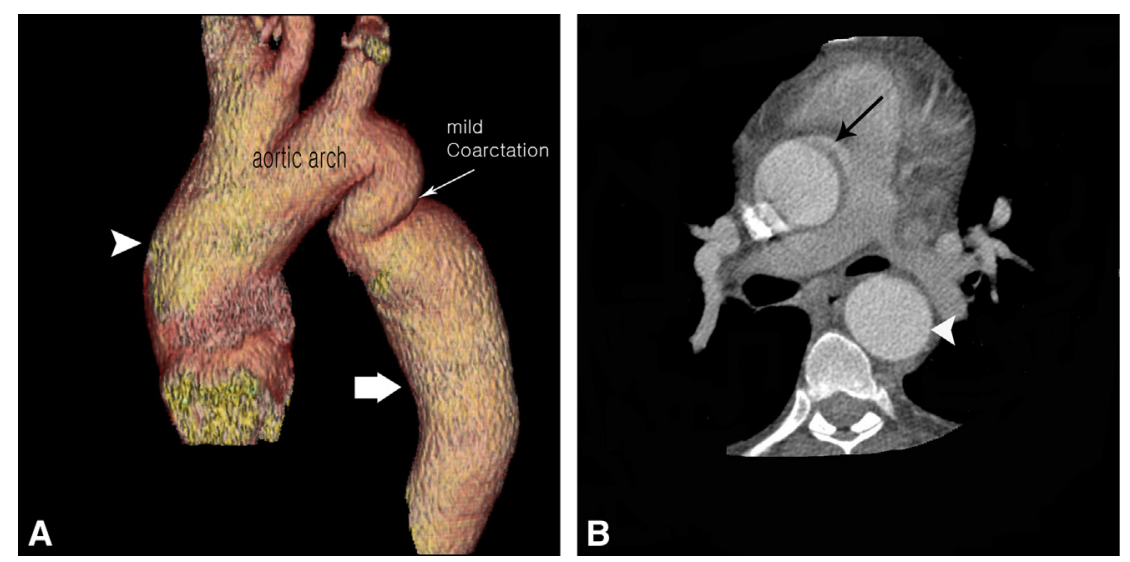

FIGURE 1. A, A 30-year-old woman with a bovine aortic arch and mild coarctation of the descending aorta (volume-rendered image) that does not require intervention. White arrowhead shows ascending aorta, curved grey arrow shows brachiocephalic artery, thin grey arrow shows the left common carotid artery that shares a common origin with the brachiocephalic artery, grey arrowhead shows the left subclavian artery, thin white arrow shows site of coarctation, and fat white arrow shows descending aorta. No significant collaterals were present. B, Axial view of (A), which shows incidental finding of pseudo-dissection of ascending aorta (thin black arrow) that does not require any intervention. White arrowhead shows descending aorta. This finding of pseudo-dissection emphasizes the importance of gating with CCT when evaluating for aortic dissection.

intracerebral aneurysm. The cause usually is congenital but sometimes acquired (as with inflammatory diseases of the aorta, eg, Takayasu arteritis or, rarely, severe atherosclerosis). ${ }^{6}$
Diagnosis is typically established with noninvasive methods, such as cardiac magnetic resonance, echocardiography, and CCT. Cardiac catheterization is indicated when associated coronary artery disease is suspected and surgery
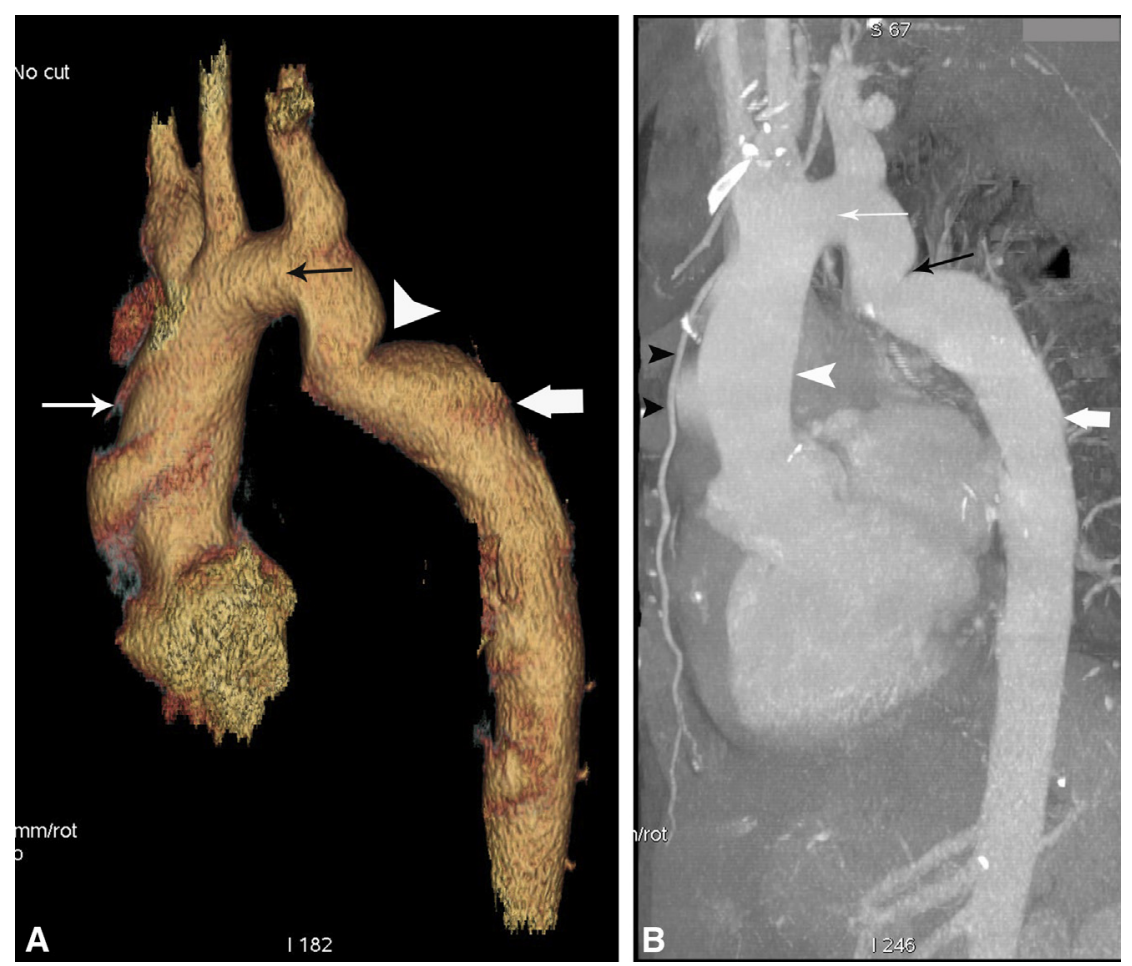

FIGURE 2. A, A 40-year-old man with coarctation of the descending aorta (volume-rendered image) amenable to percutaneous intervention. Thin white arrow shows ascending aorta, thin black arrow shows aortic arch, white arrowhead shows site of coarctation, and fat white arrow shows descending aorta. B, Sagittal image of (A). Black arrowheads show markedly dilated right internal thoracic artery (acting as collateral vessel), white arrowhead shows ascending aorta, thin white arrow shows aortic arch, thin black arrow at coarctation site, and fat white arrow at descending aorta. 

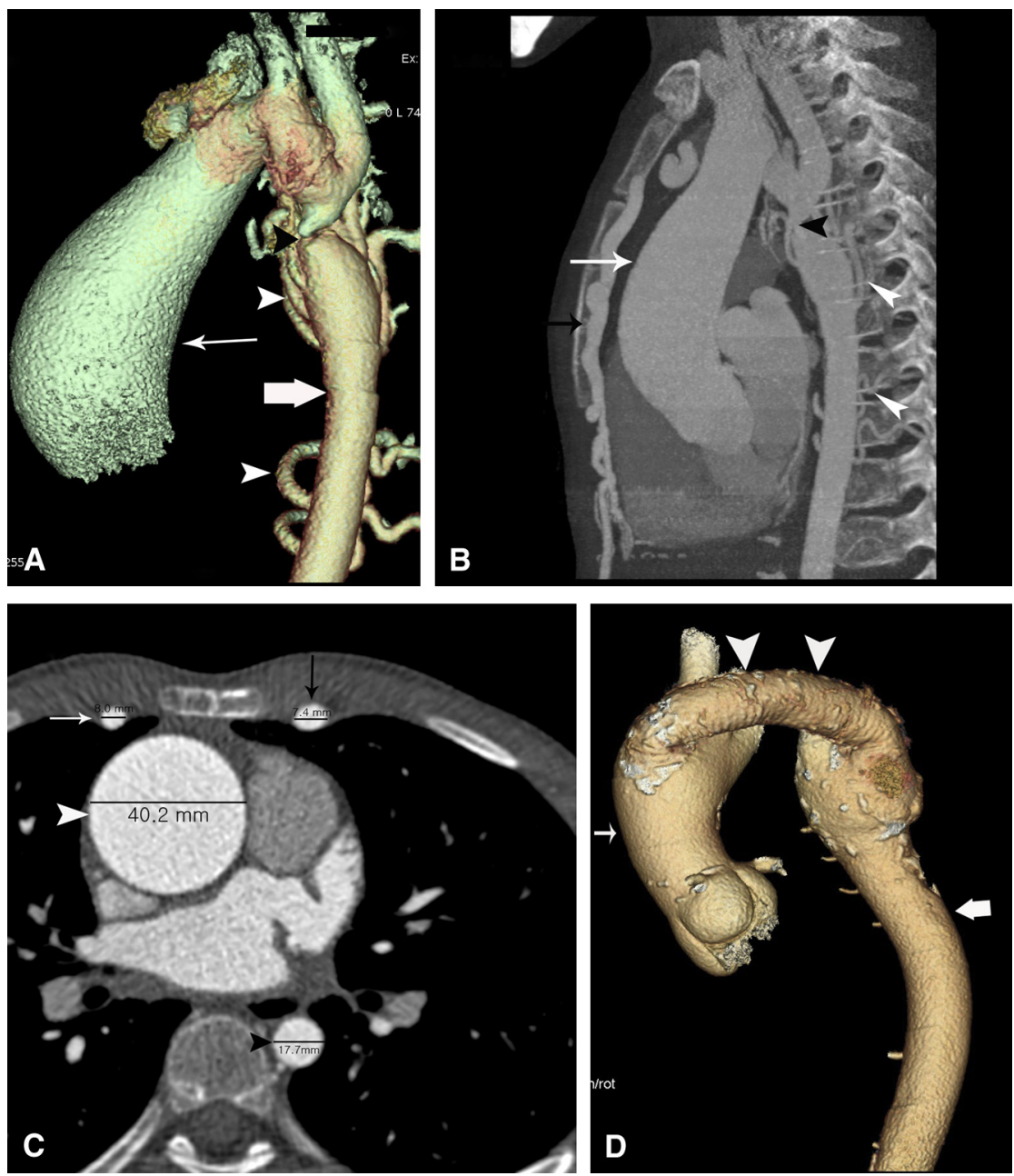

FIGURE 3. A-C, An 11-year-old boy with total coarctation at the descending aorta origin with huge collaterals, amenable to surgical correction. A, Volume-rendered image with thin white arrow shows dilated ascending aorta, black arrowhead shows site of coarctation, white arrowheads show large collaterals, and fat white arrow shows descending aorta. B, Sagittal view of (A). Thin black arrow shows dilated RIMA, thin white arrow shows dilated ascending aorta, black arrowhead shows coarctation site, and white arrowheads show huge collaterals. C, Axial view of (A). Thin white arrow shows dilated RIMA, thin black arrow shows dilated LIMA, white arrowhead shows dilated ascending aorta, and black arrowhead shows descending aorta. D, Volume-rendered image of 62-year-old man after surgical coarctation repair. Thin white arrow shows ascending aorta, white arrowheads show conduit (graft), and fat white arrow shows descending aorta.

is planned or if catheter-based intervention is to be performed.

\section{MANAGEMENT}

The 2008 American College of Cardiology/American Heart Association guidelines for adults with congenital heart disease recommend intervention for coarctation in the following circumstances ${ }^{7}$ : (1) peak-to-peak coarctation gradient of $20 \mathrm{~mm} \mathrm{Hg}$ or greater and (2) peak-topeak coarctation gradient less than $20 \mathrm{~mm} \mathrm{Hg}$ in the presence of anatomic imaging evidence of significant coarctation with radiologic evidence of significant collateral flow.

We present 4 types of patients with coarctation of the aorta with different management options: patients who do not require any intervention or procedure (Figure 1); patients with coarctation that is amenable to percutaneous intervention (balloon angioplasty with or without stent placement) (Figure 2); patients with coarctation that is amenable to surgical repair (types of surgical repair include resection with end-to-end anastomosis, subclavian flap aortoplasty, and bypass graft across area of coarctation) (Figure 3); and patients with coarctation not amenable to percutaneous or surgical intervention (Figure 4).

\section{CONCLUSIONS}

Decreasing doses of $\mathrm{CCT}^{8,9}$ and high spatial resolution combine to provide accurate assessment of the severity, 

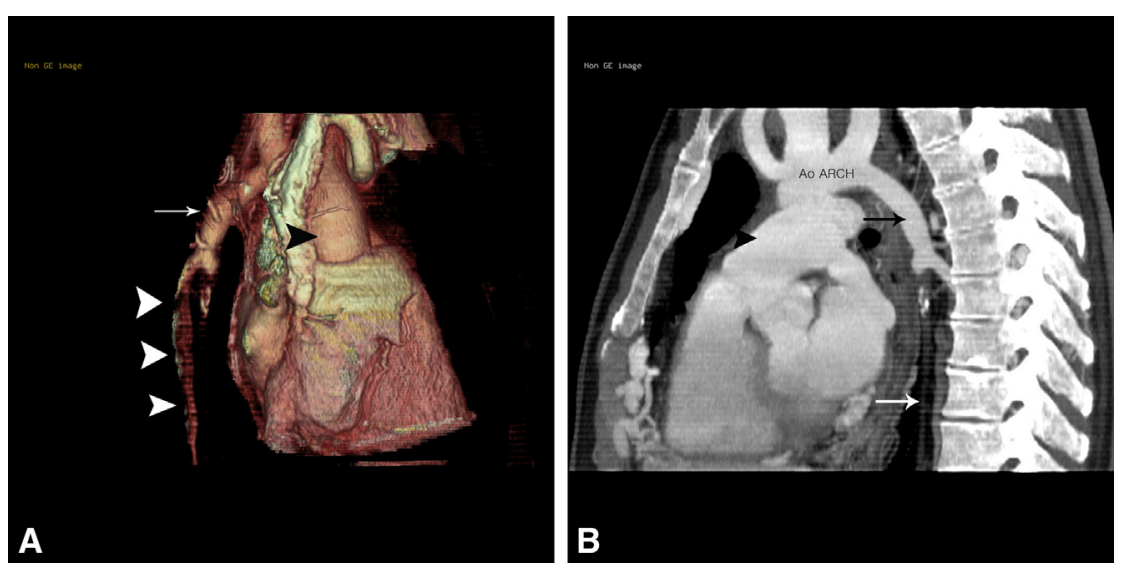

FIGURE 4. A 33-year-old woman with total interruption of the descending thoracic aorta at the T4 level with atretic (obliterated) descending aorta distal to interruption. This is not amenable to percutaneous or surgical repair. The aorta reconstituted in the abdomen at the L1 level before the origin of renal arteries. A, Volume-rendered image with black arrowhead shows ascending aorta, thin white arrow shows proximal portion of descending aorta, and white arrowheads show atretic portion of descending aorta. B, Sagittal image of (A). Black arrowhead shows ascending aorta, thin black arrow shows proximal portion of descending thoracic aorta, and thin white arrow shows atretic portion of the descending aorta.

location, and other abnormalities associated with coarctation of the aorta.

\section{References}

1. Gopal A, Loewinger L, Budoff MJ. Aortic coarctation by cardiovascular CT angiography. Catheter Cardiovasc Interv. 2010;76:551-2.

2. Budoff MJ. Maximizing dose reductions with cardiac CT. Int J Cardiovasc Imaging. 2009;25:279-87.

3. Budoff MJ. Aortic, renal and carotid CT angiography. In: Budoff M, Shinbane J, eds. Cardiac CT imaging: diagnosis of cardiovascular disease. Ed 1. London: Springer; 2006:207-22.

4. Nihoyannopoulos P, Karas S, Sapsford RN, et al. Accuracy of two-dimensional echocardiography in the diagnosis of aortic arch obstruction. J Am Coll Cardiol. 1987; 10:1072.

5. Levine JC, Sanders SP, Colan SD, et al. The risk of having additional obstructive lesions in neonatal coarctation of the aorta. Cardiol Young. 2001;11:44.
6. Sheikhzadeh A, Giannitsis E, Gehl HB, et al. Acquired thromboartheromatous coarctation of the aorta: acquired coarctation of the aorta. Int J Cardiol. 1999; 69:87.

7. Warnes CA, Williams RG, Bashore TM, et al. ACC/AHA 2008 Guidelines for the Management of Adults with Congenital Heart Disease: a report of the American College of Cardiology/American Heart Association Task Force on Practice Guidelines (writing committee to develop guidelines on the management of adults with congenital heart disease). Circulation. 2008;118: e714.

8. Gopal A, Mao SS, Karlsberg D, et al. Radiation reduction with prospective ECGtriggering acquisition using 64-multidetector computed tomographic angiography. Int J Cardiovasc Imaging. 2009;25:405-16.

9. Mark DB, Berman DS, Budoff MJ, et al. ACCF/ACR/AHA/NASCI/SAIP/ SCAI/SCCT 2010 expert consensus document on coronary computed tomographic angiography: a report of the American College of Cardiology Foundation Task Force on Expert Consensus Documents. J Am Coll Cardiol. 2010;55: 2663-99. 